\title{
Directed fast electron beams in ultraintense picosecond laser irradiated solid targets
}

\author{
X.L. Ge ${ }^{1,2,8}$, X.X. $\operatorname{Lin}^{3}$, X.H. Yuan ${ }^{1,2,4, *}$, D.C. Carroll ${ }^{4,6}$, R.J. Gray ${ }^{4}$, T.P. Yu ${ }^{7}$, O. Tresca ${ }^{4}$, M. Chen ${ }^{1,2}$, F. \\ Liu $^{1,2}$, H.B. Zhuo ${ }^{7,2}$, B. Zielbauer ${ }^{5}$, L. Zhao ${ }^{8}$, D. Neely ${ }^{6,4}$, Z.M. Sheng ${ }^{1,2,4}$, Y.T. Li ${ }^{3,2, *}$ and P. McKenna ${ }^{4}$ \\ ${ }^{1}$ Key Laboratory for Laser Plasmas (MoE) and Department of Physics and Astronomy, \\ Shanghai Jiao Tong University, Shanghai 200240, China \\ ${ }^{2}$ IFSA Collaborative Innovation Center, Shanghai Jiao Tong University, Shanghai 200240, China \\ ${ }^{3}$ Beijing National Laboratory of Condensed Matter Physics, \\ Institute of Physics, Chinese Academy of Sciences, Beijing 100190, China \\ ${ }^{4}$ SUPA Department of Physics, University of Strathclyde, Glasgow G4 ONG, United Kingdom \\ ${ }^{5}$ PHELIX Group, Gesellschaft für Schwerionenforschung mbH, Planckstrasse 1, D-64291,Darmstadt, Germany \\ ${ }^{6}$ Central Laser Facility, STFC Rutherford Appleton Laboratory, Oxfordshire OX11 0QX, United Kingdom \\ ${ }^{7}$ College of Science, National University of Defense Technology, Changsha 410073, China and \\ ${ }^{8}$ State Key Laboratory of Surface Physics and Department of Physics, Fudan University, Shanghai 200433, China*
}

(Dated: August 13, 2015)

\begin{abstract}
We report on fast electron transport and emission patterns from solid targets irradiated by s-polarized, relativistically intense, picosecond laser pulses. A beam of multi-MeV electrons is found to be transported along the target surface in the laser polarization direction. The spatial-intensity and energy distributions of this beam is compared with the beam produced along the laser propagation axis. It is shown that even for peak laser intensities an order of magnitude higher than the relativistic threshold, laser polarization still plays an important role in electron energy transport. Results from 3D particle-in-cell simulations confirm the findings. The characterization of directional beam emission is important for applications requiring efficient energy transfer, including secondary photon and ion source development.
\end{abstract}

PACS numbers: 52.38.Kd, 41.75.Jv, 52.38.-r

The transport of energetic ('fast') electrons in dense plasma produced by short, high-intensity laser pulses is important to the optimization of laser-driven ion beams $[1,2]$, to the production of ultrafast novel radiation sources [3-5] and to the development of the fast ignition (FI) approach to inertial confinement fusion (ICF) [6]. These applications require efficient laser energy coupling to directed beams of fast electrons. For example, target normal sheath acceleration (TNSA) [7], the dominant target-rear-side ion acceleration mechanism for relativistic laser intensities $\left(I>10^{18} \mathrm{~W} / \mathrm{cm}^{2}\right.$ for $\sim 1 \mu \mathrm{m}$ wavelength light) and micron-thick targets benefits from efficient laser energy transfer into large currents of forward-directed multi-MeV electrons at the target front side. These fast electrons propagate into the target and produce a strong electrostatic sheath field upon escaping at the rear, leading to ionization and the acceleration of the resulting ions to multi-MeV energies. It has been shown that lateral spreading of fast electrons along the target surfaces also occurs [8-10], and that this can reduce the energy coupling to the TNSA-ions. This charge spreading is either isotropic across the target surface due to fast electrons refluxing within the target $[11,12]$, or anisotropic in the incident plane due to their confinement by the self-generated electrostatic fields formed on the surface $[8,13,14]$. The anisotropically distributed fast electrons along the target surface affect the shape of the sheath field and thus the ion beam distribution [12].

In previous work [15], at a non-relativistic intensity of $2 \times 10^{16} \mathrm{~W} / \mathrm{cm}^{2}$, collimated beams of fast electrons in the polarization direction were observed with $s$-polarized laser light

\footnotetext{
*Email Address: xiaohui.yuan@sjtu.edu.cn; ytli@iphy.ac.cn
}

(i.e. with the laser polarization vector directed along the target surface). At higher laser intensity $I \sim 10^{18} \mathrm{~W} / \mathrm{cm}^{2}$, Li et al [16] reported the effects of laser polarization on fast electron and proton beam emission in the laser irradiation plane using 70 fs laser pulses. However, to date the role of polarization on the lateral transport of fast electrons at the relativistic laser intensities required for applications such as ion acceleration and FI has not been explored.

In this Letter, we present spatially resolved measurements of fast electron emission patterns from solids irradiated by picosecond laser pulses with peak intensity up to $1.8 \times 10^{19}$ $\mathrm{W} / \mathrm{cm}^{2}$. It is shown that, in addition the electron beam injected into the target along the laser axis, a relatively lowdivergence beam of fast electrons is also directed along the target surface, in the laser polarization direction. Full 3dimensional particle-in-cell (PIC) simulations qualitatively reproduce the experiment results. It is thus shown that even at a peak laser intensity more than one order of magnitude higher than the relativistic threshold, the polarization of the laser beam continues to strongly influence fast electron energy transport.

The experiment was performed using the PHELIX laser at GSI in Darmstadt, Germany. The laser pulses were $s$ polarized, with $0.5 \mathrm{ps}$ duration at full width at half maximum (FWHM), and $1.054 \mu \mathrm{m}$ central wavelength. The pulses, with energy up to $100 \mathrm{~J}$, were focused using an $f / 9$ off-axis parabolic mirror to a focal spot of $18 \mu \mathrm{m} \times 27 \mu \mathrm{m}$ (FWHM, with the major axis in vertical direction, containing $35 \%$ of laser energy) on target, giving the calculated peak intensity of $1.8 \times 10^{19} \mathrm{~W} / \mathrm{cm}^{2}$. In addition, for some shots, the laser pulse energy was set to $\sim 10 \mathrm{~J}$, producing an intensity of $1.8 \times 10^{18} \mathrm{~W} / \mathrm{cm}^{2}$ which is just above the relativistic threshold (for $1.054 \mu \mathrm{m}$ light). The beam was incident at $45^{\circ}$ with 


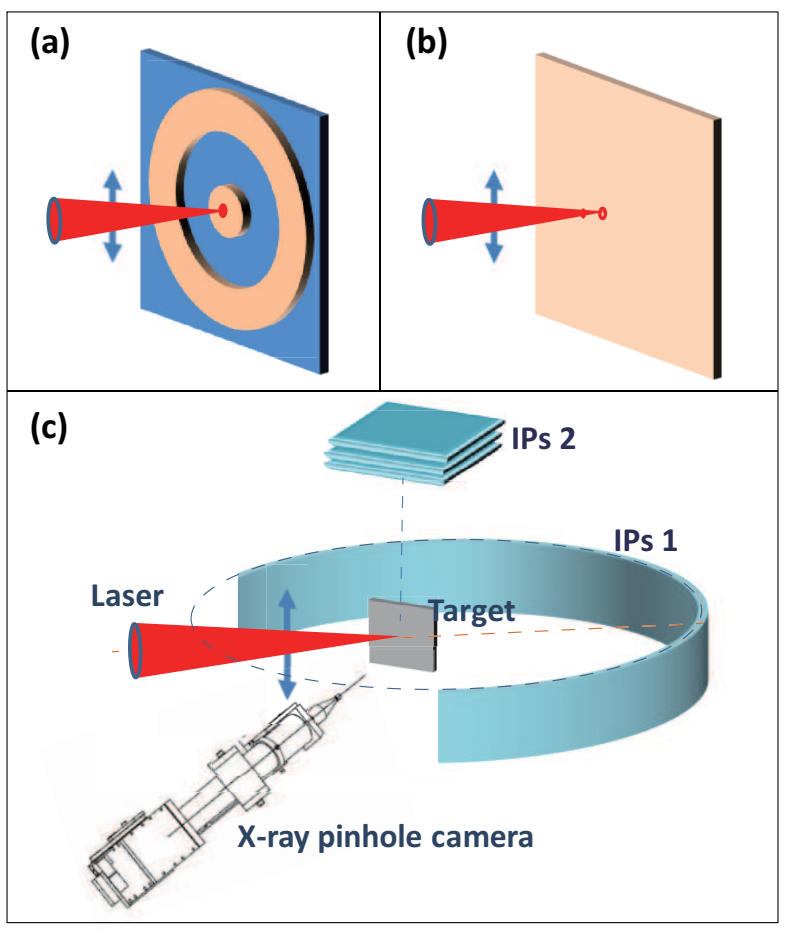

FIG. 1. (Color online). (a) Patterned target consisting of a Cu layer (in orange) on an insulator substrate (in blue). (b) Uniform $\mathrm{Cu}$ or $\mathrm{Cu} / \mathrm{Ni}$ alloy foil used as a reference target. (c) Schematic layout showing positions of image plates stacks ('IPs 1' and 'IPs 2') and $\mathrm{x}$-ray pinhole camera. The laser polarization direction is marked by blue arrows.

respect to the target normal, in the horizontal plane. The measured intensity contrast was $\sim 10^{7}$ at $3 \mathrm{~ns}$ and $\sim 10^{6}$ up to $50 \mathrm{ps,}$ prior to the peak of the pulse.

The primary target consisted of a patterned, $20 \mu \mathrm{m}$-thick, $\mathrm{Cu}$ layer on a $1 \mathrm{~mm}$-thick plastic substrate, as shown schematically in Fig. 1(a). The central $\mathrm{Cu}$ disk had a diameter of $60 \mu \mathrm{m}$ and the concentric ring had an inner and outer diameter of $320 \mu \mathrm{m}$ and $440 \mu \mathrm{m}$, respectively. Planar, unpatterned foils of $\mathrm{Cu}$ and $\mathrm{Cu} / \mathrm{Ni}$ alloy (Fig. 1(b)), both $20 \mu \mathrm{m}$ thick, were used as reference targets [14].

A pinhole camera was used to image the x-ray emission produced at the front side of the target. The camera was positioned at $10^{\circ}$ to the target normal axis in the plane of laser irradiation. The pinhole was $10 \mu \mathrm{m}$ in diameter, positioned at a distance of $35 \mathrm{~mm}$ from the target and $379 \mathrm{~mm}$ from the image plate (IP, Fujifilm BAS-SR type) detector. The IP was filtered with $13 \mu \mathrm{m}$ aluminum foil to shield it from the laser light, which effectively sets the minimum measurable x-ray threshold energy to $1 \mathrm{keV}$. A stacked detector ('IPs 1'), consisting of 4 layers of IP (BAS-MP), was positioned surrounding the target at a radius of $65 \mathrm{~mm}$, covering an arc of $230^{\circ}$ in the horizontal plane. For practical reasons, the stack was positioned to sample the lower half of the electron distribution emitted from the target.

Another stack ('IPs 2') with eight layers of IP (BAS-MP) of $50 \mathrm{~mm} \times 50 \mathrm{~mm}$ in size was positioned above the target,

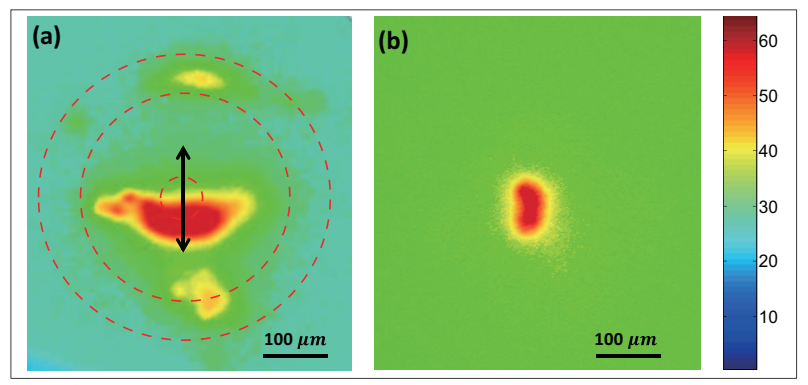

FIG. 2. (Color online). Example x-ray images measured with the pinhole camera, for (a) the patterned target, and (b) a reference target. The arrow in (a) indicates the laser polarization direction in both examples. Dashed circles show the initial positions of the disk and the concentric ring. Both images are comparable in terms of color scale, which has been chosen to highlight that the $\mathrm{x}$-ray emission from the ring is primarily directed along the polarization axis.

at $29 \mathrm{~mm}$ from the laser focus. $1 \mathrm{~mm}$-thick iron filters were placed before each IP layer (several filters used between some layers) in both stacks. Each stack was further wrapped with aluminum foil to avoid direct exposure to optical light. The stack arrangements enable measurement of the angular distribution of the escaped fast electrons with high resolution both in the horizontal plane and in the laser polarization direction. Fig. 1(c) shows a schematic of the experiment arrangement.

The x-ray measurement from the patterned target is shown in Fig. 2(a) for an intensity of $1.8 \times 10^{19} \mathrm{~W} / \mathrm{cm}^{2}$. Three $\mathrm{x}$ ray sources across the target are observed. The strongest signal is from the central disk and is asymmetrically distributed. The central spot is elongated in the horizontal plane with a horizon-to-vertical ratio of 2.5:1. This orientation is perpendicular to the major axis of the laser focus. In comparison, we see only a single elliptical $\mathrm{x}$-ray hot spot of $60 \mu \mathrm{m} \times 100 \mu \mathrm{m}$ FWHM from the reference $\mathrm{Cu} / \mathrm{Ni}$ target at similar laser intensities. A typical image is shown in Fig. 2(b). In this case, the orientation of the ellipse is parallel to the major axis of the laser focal spot. The size of the X-ray region is $42 \mu \mathrm{m}$ and $73 \mu \mathrm{m}$ in minor and major axes, respectively. The measurements indicate that the lateral spreading of the electron current is mainly directed in the direction of the laser polarization for the reference target, but perpendicular to the polarization for the patterned target for which the transverse size of the $\mathrm{Cu}$ region is limited. This change in orientation of the $\mathrm{X}$ ray emission is attributed to an anisotropic lateral expansion of the small ('mass-limited') Cu plasma driven by the oblique angle of the laser incidence in the horizontal plane. The peak intensity of the $\mathrm{x}$-ray signal from the patterned target is about two times higher than the corresponding signal from the reference target. The integrated $\mathrm{x}$-ray flux on the other hand is only slightly higher.

In addition to the central spot, $\mathrm{x}$-ray emission is also measured directly above and below in the region corresponding to the outer $\mathrm{Cu}$ ring, as shown in Fig. 2(a). The peak intensity of the $\mathrm{x}$-ray signal in these larger radii sources is about one order smaller than that in the central source. These two sources are along the laser polarization direction, indicating a preferential 


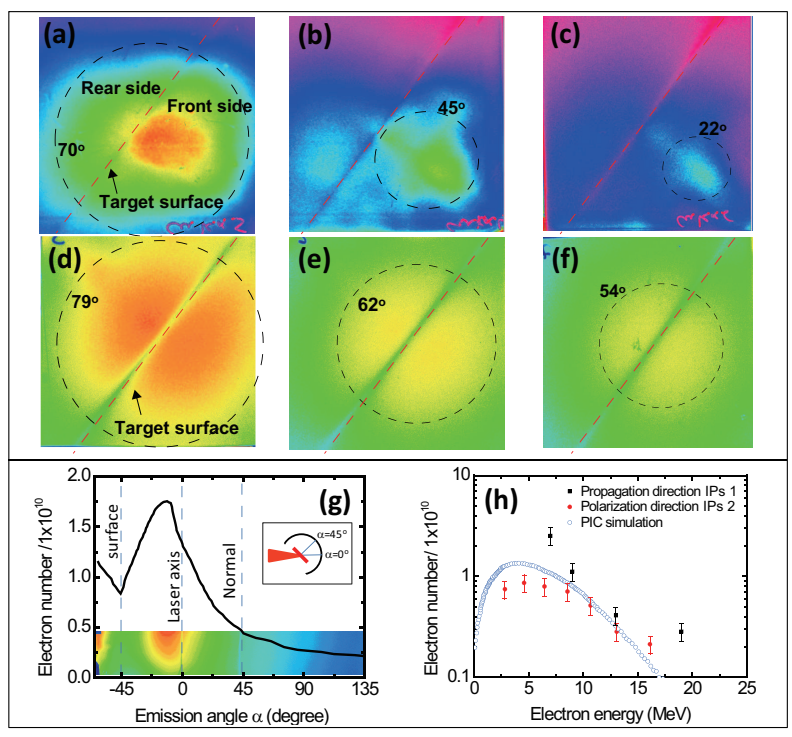

FIG. 3. (Color online). (a)-(c) Spatial-intensity distribution of the fast electrons measured by 'IPs 2' for the patterned target irradiated at $\mathrm{I}=1.8 \times 10^{19} \mathrm{~W} / \mathrm{cm}^{2}$, for electron energies above: (a) $6.4 \mathrm{MeV}$; (b) $8.5 \mathrm{MeV}$; (c) $10.7 \mathrm{MeV}$. (d)-(f) Corresponding measurements for the reference target irradiated at $\mathrm{I}=1.8 \times 10^{18} \mathrm{~W} / \mathrm{cm}^{2}$. The red dashed line marks the target surface. The circles mark example (given) divergence angles. (g) Example spatial-intensity profile and angular distribution of the fast electrons in the plane of the laser incidence (lower half of the distribution, as measured using 'IPs 1'). The inset shows how the angles are defined. (h) Energy spectra of the fast electron beams. The blue squares correspond to the laser propagation direction (from 'IPs 1' ) and red dots are for the polarization direction (from 'IPs 2'). The blue circles are from 3-D PIC simulation, for the polarization direction.

direction for the fast electrons at large radii from the source.

To investigate this observation further the fast electrons escaping the patterned target in the upward (vertical) direction were measured. Example spatial-intensity distributions are shown in Fig. 3(a-c) at three different electron lower energy thresholds. The thresholds arise due to electron stopping in the filters between the IPs in the stack, and were calculated using the continuous-slowing-down-approximation (CDSA) range table from the ESTAR database [17]. A clear beam profile is observed, for which the divergence decreases with increasing electron energy. It is likely that a similar beam is directed downward, but this could not be tested due to practical constraints arising from the target mount and drive system used. The upward-directed fast electrons account for the measured bremsstrahlung $\mathrm{x}$-ray emission produced in the $\mathrm{Cu}$ ring in the patterned target (Fig. 2(a)).

A similar measurement was made with the reference target, which also produces a vertically-directed electron beam. Fig. 3(d-f) shows an example measurement with the reference target at an order of magnitude lower peak laser intensity $\left(\mathrm{I}=1.8 \times 10^{18} \mathrm{~W} / \mathrm{cm}^{2}\right)$. The electron current is approximately symmetric about the target surface.

The vertically-directed (polarization-driven) surface elec-

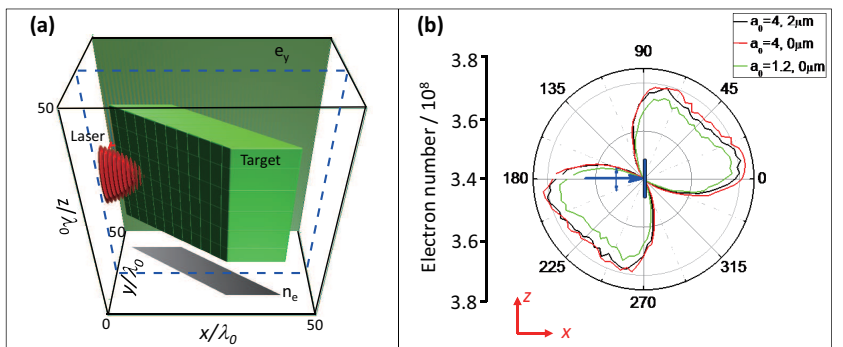

FIG. 4. (Color online) (a) Output of the simulation model at $t=10 T_{0}$ when the laser front just reaches the target surface. The laser propagates along the $x$-axis, centred at $y=25 \lambda_{0}$ and $z=25 \lambda_{0}$, and is polarized along the $z$-axis. The laser intensity distribution, with $a^{2}>4$, is shown in red. (b) Simulation results showing the electron angular distributions at $t=150 T_{0}$ in the $x-z$ plane $\left(y=25 \lambda_{0}\right)$, as shown by the dashed blue square in (a), for the three stated example combinations of peak laser intensity and preplasma scale lengths.

tron beam was further quantitatively characterized and compared to the horizontally-directed surface current (in the irradiation plane) and the laser axis-directed fast electrons (generated by the well-known $\mathrm{J} \times \mathrm{B}$ mechanism [18]). Fig. 3(g) shows the angular distribution and the integrated profile of the fast electrons (lower half of the beam) in the plane of the laser beam. A collimated beam, directed close to the laser axis (with a small deflection towards target surface) is observed (similar to that reported in reference [19]). Importantly, a beam is not produced along the target surface in the plane of the interaction (i.e. perpendicular to the laser polarization direction). The beam distribution at $\alpha<-55^{\circ}$ ( $\alpha$ is the angle in the laser irradiation plane, where $\alpha=0^{\circ}$ defines the laser propagation direction) is part of a collimated beam in the laser specular reflection direction. Fig. 3(h) is the energy spectra of the electron beam directed along the laser polarization and propagation directions, as obtained by integrating the signal in each of the IP layers and factoring in the detector energy response function. Both distributions exhibit a similar electron temperature, but the number of relatively low energy electrons produced in the polarization direction is significantly smaller. This is accounted for by additional stopping induced by collisions of these electrons with the target surface layer as they spread laterally along it.

Similar electron emission patterns are observed in full 3dimensional PIC simulations using the VLPL code [20, 21]. Due to the considerable computation resource required to run fully 3D PIC simulations, a simulation box of $x \times y \times z=$ $50 \mu \mathrm{m} \times 50 \mu \mathrm{m} \times 50 \mu \mathrm{m}$ is employed in the present study. This is sampled by $500 \times 500 \times 500$ cells with 8 electrons and 8 protons in each. The time step is $\Delta t=0.05 T_{0}$, where $T_{0}=\lambda_{0} / c=3.3 \mathrm{fs}$ is the laser period.

A fully ionized hydrogen target is placed in the center of the simulation box to model the solid target, which is at $45^{\circ}$ to the laser propagation direction ( $x$-axis). The plasma density ramps up linearly from 0 to $50 n_{c}$ (where $n_{c}=1.1 \times 10^{21}$ $\mathrm{cm}^{-3}$ is the critical density) over $2 \mu \mathrm{m}$ and is constant thereafter for $15 \mu \mathrm{m}$. The expected preplasma expansion profile 
in the experiment was simulated using the $2 \mathrm{D}$ hydrodynamic code POLLUX [22]. This revealed a very small scale length of $1.5 \mu \mathrm{m}$ at critical density. Given that this level of preexpansion is much smaller than the lateral extent of the laser focal spot, it is not expect to have a significant effect on the interaction physics. Nevertheless PIC simulation were performed with and without the density ramp. The target transverse length in the 3D PIC simulations is $40 \mu \mathrm{m}$. A $s$-polarized (along $z$-axis) laser pulse with a Gaussian spatio-temporal profile, $a=a_{0} \exp \left(-t^{2} / \tau_{0}^{2}\right) \exp \left(-r^{2} / \sigma_{0}^{2}\right)$, is incident from lefthand side (i.e. in the $+x$ direction) and focuses at the target front surface. Here, $a_{0}=4$ is the dimensionless maximum laser electric field amplitude, the laser pulse duration $\tau_{0}=30 T_{0}, r=\sqrt{y^{2}+z^{2}}$ and $\sigma_{0}=12 \mu \mathrm{m}$. This corresponds to a peak laser intensity equal to $2.2 \times 10^{19} \mathrm{~W} / \mathrm{cm}^{2}$, i.e. close to that in the experiment. Two sets of simulations with no preplasma are also performed for $a_{0}=4.0$ and $a_{0}=1.2$, corresponding to peak laser intensity equal to $2.2 \times 10^{19} \mathrm{~W} / \mathrm{cm}^{2}$ and $1.8 \times 10^{18} \mathrm{~W} / \mathrm{cm}^{2}$, respectively. Fig. 4 (a) shows the simulation configuration when the laser front just reaches the target at $t=10 T_{0}$ for $a_{0}=4$ and no preplasma. For simplicity, we only show the laser intensity (dark red) for amplitude $a^{2}>4$.

Although the configuration is simplified in the simulations, the measured electron dynamics are qualitatively reproduced. Fig. 3(h) and Fig. 4(b) show the simulation results at $t=$ $150 T_{0}$ when the laser-target interaction has already ceased. The spectrum of upward-directed electrons $(+z$-axis) exhibits a Maxwellian distribution. The cut-off energy is about 17 $\mathrm{MeV}$ and the mean energy is around $4 \mathrm{MeV}$, both of which are very similar to that measured experimentally, as shown in Fig. 3(h). Fig. 4(b) shows the angular distributions of fast electrons in the plane of the polarization for the three combinations of laser intensity and preplasma conditions. It shows that the profiles are weakly dependent on the laser and preplasma parameters, which qualitatively reproduce the experiment results in Fig. 2. Electrons are found to be directed both forward and backward along the laser propagation axis. The forward component corresponds to the strongest signal in the $\mathrm{x}$-ray image shown in Fig. 2(a). Electrons are also directed along the polarization direction ( $z$-axis). These results are in good agreement with the experiment measurements and qualitatively demonstrate the directional fast electron transport in the target.

In summary, we report on measurements of the directionality of fast electron currents in solids irradiated by ultraintense, picosecond laser pulses. It is found that a beam of electrons is directed along the target surface in the laser polarization axis at a peak laser intensity one-order magnitude higher than the relativistic intensity threshold. Thus, although electrons are accelerated along the laser propagation direction by the $\mathrm{J} \times \mathrm{B}$ mechanism near the peak of the laser pulse intensity profile, polarization effects are likely to dominate the direction of electrons accelerated at the lower intensities on the rising edge of the pulse. The observations are reproduced by full 3D PIC simulations. This is the first study in which this feature is investigated and the results highlight the importance of laser polarization in lateral electron energy transport and thus energy coupling to secondary radiation sources.

We gratefully acknowledge the expert support of the staff at the PHELIX laser facility. This work was supported by the National Basic Research Program of China (grant nos. 2013CBA01502), the National Natural Science Foundation of China (Grant Nos 11421064 and 11205100), EPSRC (Grants Nos EP/J003832/1, EP/K022415/1 and EP/L001357/1), and EU COST P-14 Action, LaserLAB Europe. Data associated with research published in this paper is accessible at http://dx.doi.org/10.15129/...[insert DOI].
[1] H. Daido, M. Nishiuchi and A.S. Pirozhkov, Rep. Prog. Phys. 75, 056401 (2012).

[2] A. Macchi, M. Borghesi and M. Passoni, Rev. Mod. Phys. 85, 571 (2013).

[3] U. Teubner, K. Eidmann, U. Wagner, U. Andiel, F. Pisani, G.D. Tsakiris, K. Witte, J. Meyer-ter-Vehn, T. Schlegel, and E. Förster, Phys. Rev. Lett. 92, 185001 (2004).

[4] L.M. Chen, M. Kando, M.H. Xu, Y.T. Li, J. Koga, M. Chen, H. Xu, X.H. Yuan, Q.L. Dong, Z.M. Sheng, S.V. Bulanov, Y. Kato, J. Zhang, and T. Tajima, Phys. Rev. Lett. 100, 045004 (2008).

[5] T.P. Yu, A. Pukhov, Z.M. Sheng, F. Liu, and G. Shvets, Phys. Rev. Lett. 110, 045001 (2013).

[6] M. Tabak, J. Hammer, M.E. Glinsky, W.L. Kruer, S.C. Wilks, J. Woodworth, E.M. Campbell, and M.D.Perry, Phys. Plasmas 1, 1626 (1994).

[7] S.C. Wilks, A.B. Langdon, T.E. Cowan, M. Roth, M. Singh, S. Hatchett, M.H. Key, D. Pennington, A. MacKinnon, and R. A. Snavely, Phys. Plasmas 8, 542 (2001).

[8] T. Nakamura, S. Kato, H. Nagatomo, and K. Mima, Phys. Rev. Lett. 93, 265002 (2004).

[9] Y.T. Li, X.H. Yuan, M.H. Xu, Z.Y. Zheng, Z.M. Sheng, M. Chen, Y.Y. Ma, W.X. Liang, Q.Z. Yu, Y. Zhang, F. Liu, Z.H.
Wang, Z.Y. Wei, W. Zhao, Z. Jin, and J. Zhang, Phys. Rev. Lett. 96, 165003 (2006).

[10] P. McKenna, D.C. Carroll, R.J. Clarke, R.G. Evans, K.W.D. Ledingham, F. Lindau, O. Lundh, T. McCanny, D. Neely, A.P.L. Robinson, L. Robson, P.T. Simpson, C.-G. Wahlström, and M. Zepf, Phys. Rev. Lett. 98, 145001 (2007).

[11] X.X. Lin, Y.T. Li, F. Liu, B.C. Liu, F. Du, S.J. Wang, L.M. Chen, L. Zhang, Y. Zheng, X. Liu, X.L. Liu, Z.H. Wang, J.L. Ma, Z.Y. Wei, and J. Zhang, Rev. Sci. Instrum. 82, 036104 (2011).

[12] O. Tresca, D.C. Carroll, X.H. Yuan, B. Aurand, V. Bagnoud, C.M. Brenner, M. Coury, J. Fils, R.J. Gray, T. Kühl, C. Li, Y.T. Li, X.X. Lin,M.N. Quinn, R.G. Evans, B. Zielbauer, M. Roth, D. Neely, and P. McKenna, Plasma Phys. Control. Fusion 53, 105008 (2011).

[13] M. Chen, Z.M. Sheng, J. Zheng, Y.Y. Ma, M.A. Bari, Y.T. Li, and J. Zhang, Opt. Express 14, 3093 (2006).

[14] X.H. Yuan, D.C. Carroll, J. Zheng, J.L. Liu, R.J. Gray, C.M. Brenner, M. Coury, L.M. Chen, Y. Fang, O. Tresca, B. Zielbauer,T. Kühl, Y.T. Li, D. Neely, Z.M. Sheng and P. McKenna, Plasma Phys. Control. Fusion 56, 055001 (2014).

[15] L.M. Chen, J. Zhang, Y.T. Li, H. Teng, T.J. Liang, Z.M. Sheng, 
Q.L. Dong, L.Z. Zhao, Z.Y. Wei, and X.W. Tang, Phys. Rev. Lett. 87, 225001 (2001).

[16] Z. Li, H. Daido, A. Fukumi, A. Sagisaka, K. Ogura, M. Nishiuchi, S. Orimo, Y. Hayashi, M. Mori, M. Kado, S.V. Bulanov, T. Zh. Esirkepov, Y. Oishi, T. Nayuki, T. Fujii, K. Nemoto, S. Nakamura and A. Noda, Phys. Plasmas 13, 043104 (2006). P.A. Wilson and P. McKenna, New J. Phys. 12, 063018 (2010).

[17] http://physics.nist.gov/PhysRefData/Star/Text/ESTAR.html.

[18] W.L. Kruer and K. Estabrook, Phys. Fluids 28, 430-432 (1985)

[19] R.J. Gray, X.H. Yuan, D.C. Carroll, C.M. Brenner, M. Coury,
M.N. Quinn, O. Tresca, B. Zielbauer, B. Aurand, V. Bagnoud, J. Fils, T. Kühl, X.X. Lin, C. Li, Y.T. Li, M. Roth, D. Neely, and P. McKenna, Appl. Phys. Lett. 99, 171502 (2011).

[20] A. Pukhov, J. Plasma Phys. 61, 425 (1999).

[21] T.P. Yu, L.X. Hu, Y. Yin, F.Q. Shao, H.B. Zhuo, Y.Y. Ma, X.H. Yang, W. Luo and A. Pukhov, Appl. Phys. Lett. 105, 114101 (2014).

[22] G.J. Pert, J. Comput. Phys. 43, 111 (1981). 